\title{
Routing of Vehicles from Depot in Two-Level Supply Chain with a Meta-Heuristic Algorithm
}

\author{
Zahra Sadat Hosseini ${ }^{*}$, Iman Kamali \\ 1 B.S. of Industrial Engineering, Faculty of Industrial Engineering, Sabzevar University of New Technology, Iran \\ * Corresponding author's e-mail: z.s.hosseini@gmail.com
}

\begin{abstract}
The present study aimed at routing vehicles from a depot in two-level supply chain with a meta-heuristic algorithm. This study is an analytical type of research. The issue of routing which includes time, travel duration dependent on the day, has first been introduced by Mandraki and Duskin. They proposed the issue of time-dependent Travelling Salesman Problem (TSP), such that their simplified issue was a VRP issue and service should be provided to all customers, and each customer should only be visited by one vehicle. Mathematical modeling was used as a research tool, and in the examined problem, there are a number of demand points with simultaneous delivery and return. This demand should pass the depot which should be selected from among candid points, and it should be determined that the construction of depot in each of these locations has a specific fixed cost. In order to send service from depot to the demand points, vehicles with special carrying capacity, fixed using costs, and overhead carrying costs. Given that solving the problem is time-consuming, the MOPSO meta-heuristic algorithm was used in order to solve the problem. The obtained results were fully presented, and in different repetitions it was observed that the second vehicle has the highest load carrying.
\end{abstract}

Keywords: routing, vehicles, depot, meta-heuristic algorithm

\section{INTRODUCTION}

In recent decades, our lives have improved due to advances in transportation and logistics. Transportation and logistics account for a huge percentage of total cost of any product. In fact, every company uses about $20 \%$ of total value of products and goods in the transportation and logistics sector. Therefore, the transportation sector is very important, but it has negative effects such as noise, pollution, etc. However, effective and efficient transport routing systems through computer optimization tools help to reduce the negative impacts and costs. This is because a reduction of the traversed distance helps to improve the driver and vehicle efficiency and can reduce the operational costs and greenhouse gas emissions as well as increase the customer service level. The issues of routing vehicles are diversified, including routing vehicles with limited capacity, routing vehicles with several central depots, routing vehicles with time window. Finding the routes of vehicles is a logistic issue on which many studies have recently been done, and typically, the purpose of any problem is to find a set of routes for several vehicles from a specific origin to a number of customers and returning to depot, without violating the capacity limit of any vehicle, at minimum cost. The combination of customers is not limited to the choice of transportation routes; VRP (Vehicle Routing Problem) is considered as a combinative optimization problem, in which the number of possible responses is increased along with the number of customers (Kumar et al., 2013).

Today, with the development of industries and emergence of competitive markets, managers of industrial and service centers are seeking to reduce the operational costs and thus reduce end cost of products and services. Reducing the transportation costs can be very important in saving costs. One of the issues in the field of optimizing transportation costs is actually the issue of vehicle routing. Time-dependent vehicle routing is a type of dynamic vehicle routing. This issue, in its basic 
form, is a problem of routing vehicles that are distributed in a fleet of vehicles from one or more central depots in order to serve a set of customers and, upon completion of operations, return to the relevant depot (Kok et al., 2012). The main goal of this is to minimize the total travel time and its resulting costs; under these conditions, the travel time is divided between customers as well as between customers and depot, and consequently, the travel costs are dependent on the time period during which the travel is done.

Increasing the efficiency and performance of transportation systems is one of the most important issues that need to be addressed today, so that we can face less traffic and prevent cost increase; thus, the vehicles routing problem (VRP) has emerged (Sattak et al., 2013).

Routing refers to finding an ideal route that meets the model objectives and complies with the customer, while through planning the vehicles, determines the time that each customer must be served. Accordingly, the issue of total time and cost of the route not only includes the total cost of the distance and service time and other related costs, but the total cost of stops and waiting are calculated too. Urban environments have a broad structural complexity. This has enabled access to different points through more than one route. Under such conditions, traffic regulations for routes, such as determining maximum permitted speed and traffic constraints on transportations vehicles affect the choice of the route to continue the travel (Schneider et al., 2014). Choosing the appropriate route can be very important for distribution companies and rental transportation companies, especially when receiving orders at a specific time is important for customers, and they can be optimized and selected through different algorithms. Therefore, in the present study, we will provide a model for routing the vehicles traffic with a specific origin using a meta-heuristic algorithm, and we will optimize the problem of providing a routing model.

The issue of routing vehicles was first introduced by Dantzig and Ramser in 1959. In 2001, Dethloff highlighted the applications of vehicle routing problem in simultaneous receiving and delivering of goods for the first time in reverse logistics issues, and used the innovative method of Cheapest Insertions. In 2002, Montane and Galvao defined three different types of vehicle routing problem in simultaneous receiving and delivering of goods. Salhi and Nagy in 2005 developed their previous studies. They invented an innovative method by adding more node operators which led to modification and solution of the problem. In 2006, Chen and $\mathrm{Wu}$, proposed an additive method and hybrid meta-heuristic algorithm based on a hybrid method including Tabusearch Meta-heuristic Algorithm and improvement algorithms. VRP was presented by Dantzig and Ramser in 1959 and is an important issue in transportation, distribution and logistics. VRP often includes the transfer of goods located at a central station to customers who have ordered these goods. The goal of VRP is to minimize total route cost in transporting goods from central station to customers. Determining the optimal solution is considered a full-NP problem in hybrid optimization and many definitive and heuristic practical methods were developed to find acceptable responses to VRP. Sargardan et al. (2015) presented a study entitled "Modeling Vehicle Routing Problem", considering multiple depots, simultaneous delivery and loading, hard and soft time window, cost and depreciation dependent on the amount of load existing on the vehicle and type of route. In 2016, Rahmani et al. presented a study entitled "Using Improved Gravity Algorithm for Solving Open Vehicle Routing Problem". Salamatbakhsh et al. in 2016 present a study titled "Open Vehicle Routing Problem" considering drivers' satisfaction: multi-objective evolutionary algorithm based on decomposition. In this research, the quality of MOEA/D algorithm responses in the average fleet number is about $16 \%$ better than MOPSO algorithm which indicates appropriate performance of the proposed algorithm. Hashimoto et al. (2008) designed the models based on predetermined possible routes and used solutions based on repetitive local search algorithm in order to solve it. Woensel et al. (2008) considered traffic density factor in TDVRP (Time-dependent vehicle route planning) problem and used queuing theory in order to solve it. Wang and Wang (2009) used a two-step innovative method in order to solve the vehicle routing problem with a return load in which the zero speed is dependent on time. Soler et al. (2009) transformed a vehicle routing problem with time windows which include time and costs of time-dependent travel, in several steps into a symmetric capacity-having vehicle routing problem, and solved it using the existing methods. Kuo et al. (2009) employed an optimization method based on no-search for the 
issue of goods allocation and time-dependent vehicle routing problem. Kuo (2010) examined the vehicle routing problem aiming at minimizing fuel consumption and used simulated annealing in order to solve it. Kok et al. (2010) considered work time manual for drivers in TDVRP, and solved it through an innovative method based on dynamic programming. Figliozzi (2010) studied the impacts of traffic density on the characteristics and costs of vehicle travel. Chen et al. (2006) examined the above-mentioned issue by considering time windows and urgent demands. Bock (2010) combined real-time vehicle routing problem with hybrid transportation and the possibility of multiple tranfers between various transportation vehicles. An object-oriented evolutionary framework was also presented by Liao and $\mathrm{Hu}$ (2011) for this category of issues. Liorini et al. (2011) developed a solution method for real-time vehicle routing problem, considering drivers and central distribution office.

\section{MATHEMATICAL MODELING}

The set $\mathrm{V}=\{2,3, \ldots, \mathrm{n}\}$ represents the cities to be passed. The central station, from which the travel should be started and ended, is considered as dual. The origin station and the destination station are known as 1 and $n+1$, respectively. Therefore, the tour is turned into a Hamiltonian route which starts at 1 and ends in $n+1$. Hamiltonian route is a route in which exactly one crossing occurs from each node. For convenience, we define the two sets of $V^{0}=V \cup\{1\}$ and $V^{d}=V \cup\{n+1\}$. For each pair of $\mathrm{j}$ and $\mathrm{i}$ of the cities, $t_{i j}$ represents the travel time and $C_{i j}$ represents the travel cost (distance) from city $i$ to city j. The interval $\left[a_{i}, b_{i}\right]$ represents time window of city $i$. Two important sets of variables are: the set of $\left\{S_{i} \mid i=1, \ldots, n\right\}$ in which $S_{i} \in\{2, \ldots, n+1\}$ represents cities immediately after the city i on the path, and the set $\left\{T_{i} \mid i=1, \ldots, n\right\}$ in which $T_{i}$ represents the start time of service in city $i$. A possible tour assigns a specific $\mathrm{S}$ to each city and prevents partial round. As long as the values are assigned to $S_{i}$, direct partial routes are formed in line with them. $\beta_{i}$ and $\varepsilon_{i}$ represent the beginning and end of the current partial route along $i$ that initially $\beta_{i}=\varepsilon_{i}=i$, respectively.
The target function:

$$
\min \sum_{i \in V^{o}} c_{i, s_{i}}
$$

That tries to minimize total cost of the tour. $c_{i}, s_{i}$ indicates the travel cost from i to $S_{i}$. Model constraints include:

Coordination limits:

$$
\begin{array}{ll}
S_{i} \neq S_{j} & \forall i, j \in V^{o}, i \neq j \\
S_{i} \in\{2, \ldots, n+1\} & \forall i \in V^{o}=\{1,2, \ldots, n\}
\end{array}
$$

In order to ensure that every city is passed exactly once.

Limitations of partial round removal:

$$
\begin{gathered}
S_{i} \neq i \quad \forall i \in V^{o} \\
S_{i}=j \Rightarrow S_{\varepsilon_{j}} \neq \beta_{i} \\
\forall i \in V^{d}=\{2,3, \ldots, n, n+1\}
\end{gathered}
$$

So far, the model represents a TSP problem. In order to involve time windows in the model and transform the model into a standard model for TSPTW problem, time scheduling constraints are added:

$$
\begin{gathered}
a_{i} \leq T_{i} \leq b_{i} \\
\forall i \in V^{d}=\{2,3, \ldots, n, n+1\} \\
S_{i}=j \Rightarrow T_{i}+t_{i j} \leq T_{j} \quad \forall i \in V^{o}
\end{gathered}
$$

In order to limit the service time to the related time window and to ensure the possibility of time scheduling, a temporary restriction is imposed on each arch located in the route.

In order to convert the model from TSPTW to TSPmTW, among all main constraints of the model (1 to 7), only Equation (6) requires some changes. Limitations can be combined through logical operators such as "and" $(\Lambda)$, "or" $(V)$, "negative (other than)" ( $\neg)$. Non-continuity in the time window, by adding the constraint $\left(6^{\prime}\right)$ to the original model can be expressed as follows:

$$
\bigwedge_{k=1}^{m_{i}} \neg\left(a_{i}^{k}<T_{i}<b_{i}^{k}\right) \quad \forall i \in V
$$

In which $m_{i}$ represents the number of empty time intervals of the time window of city i, meaning that $] a_{i}^{k}, b_{i}^{k}\left[\right.$ shows the $\mathrm{K}^{\text {th }}$ empty interval. For example, the city i may have a visiting possibility from 8:00 to 12:00 and from 13:00 to 17:00. The limitation of this model can be expressed as follows:

$$
\left(9 \leq T_{i} \leq 17\right)^{\wedge} \neg\left(12<T_{i}<13\right)
$$




\section{METHODOLOGY}

We will optimize the issue of providing a model for vehicle routing through an improved meta-heuristic algorithm. This optimization and presentation of the simulation results will be analyzed by means of MATLAB software. The issue of vehicle routing with specific origin will be done through meta-heuristic algorithm of ingredients congestion (PSO). First, this method is described and then, the framework and research method are described.

PSO algorithm stages are as follows:

- Initial valuation: assigning initial value to a population of particles with random positions and velocities in $\mathrm{D}$ dimensions in the search space

- Estimation: estimation of appropriateness of each particle in this population

- Update: calculation of the speed of each particle with moving to the next position

- End: stopping the algorithm if it reaches a specified stopping criterion, and otherwise, going to the estimation stage

Details of the combined PSO algorithm:

- P: PSO population size which refers to the number of sections of the training course proposed by the problem.

- $t$ : the number of professors

- Pso [i]: refers to the position of particle $i$ in PSO population, as a $p$ vector, the values of which belong to the set $(1,2, \ldots, 40 * \mathrm{~m})$

- Pso [i] [j]: represents the number of starting time intervals attributed to the $j^{\text {th }}$ section of the training course in the $i^{\text {th }}$ particle

- Fitness [i]: represents appropriateness of particle $\mathrm{i}$ based on equation (1)

- Pbest [i]: the best local position for particle i.

- Gbest [i]: The best general coefficient

- Pbest-Fitness [i]: represents the best local appropriateness of particle i.

- V [i]: velocity of particle $i$, which is a p-dimensional encoded real vector

Pso starts to work with a group of random responses (particles), then seeks to find the optimal response in the problem space through updating the particle location. Each particle is characterized by two values of $X_{i d}$ and $V_{i d}$ that indicate spatial position and speed of the $\mathrm{d}^{\text {th }}$ dimension of $i^{\text {th }}$ particle, respectively. At each stage of population movement, the location of each particle is updated with two values of the best fit the value ever obtained for each particle, separately (p-best), and the best value ever obtained by all particles in the population (g-best). Then, in each repetition, the algorithm, after finding the two values of p-best and g-best, updates the new velocity and location of each particle according to the following formulas (Yo hu yee, 2001).

$$
\begin{aligned}
& V_{i d}(t+1)=\omega V_{i d}(t)+c_{1} r\left(\text { pbest }_{i d}-x_{i d}\right)+ \\
& +c_{2} r_{2}\left(\text { Gbest }_{i d}-x_{i d}\right) \\
& X_{i d}(t+1)=X_{i d}(t)+V_{i d}(t+1)
\end{aligned}
$$

In this equation, parameters are introduced as follows:

- $\omega$ : coefficient of inertia

- $C_{1}$ and $C_{2}$ are fixed positive numbers that are related to the local and global parameters, respectively.

- $R_{1}$ and $R_{2}$ are random numbers with normal distribution in the interval [0 and 1].

The meta-heuristic algorithms, including pso, determine the decision variables in a way that firstly - these variables are within the defined range, and secondly, they minimize a specific cost function. In this research, our cost function is the sum of several cost functions, and this is why it is referred to as multi-objective optimization. Our cost function in this research includes minimizing time and cost and maximizing efficiency. In other words, routing is obtained through the lowest time and cost and highest efficiency. If the vehicle starts to move from a specific point of origin, after spending the least possible time and cost and after passing the route, it returns to the same point.

\section{Running the algorithm}

In the table below, the input intended for the customer, the depot and ... with the related coordinates are shown. In this study, we

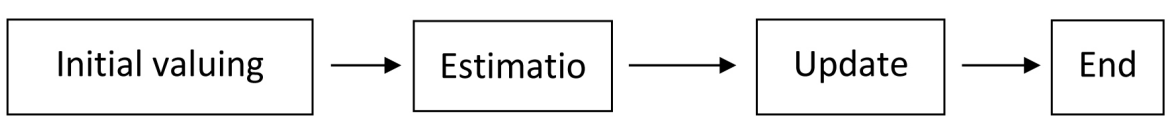

Figure 1. PSO algorithm stages 
considered 10 customers, but a greater number can be considered, too.

In the tables above, the depot coordinates are shown with $\mathrm{x}=55$ and $\mathrm{y}=25$, and the rest of coordinates related to customers can also be seen. The demand for each customer is also shown. We considered three vehicles to serve the customers, and the capacity of each one is specified.

\section{RESULTS}

After simulation, the following results were presented for three repetitions. All the three repetitions are infeasible and no practical state can be considered for them.

In the first repetition, one out of three vehicles has never been involved. The second vehicle has also passed a route about four times more than the other car, which indicates uneven division of loads between vehicles. In the second case, similarly to the first case, one vehicle has been idle and two other vehicles have passed a distance a little close to each other; however, they also have infeasible state. The third state performed better than the other two; however, its infeasible result cannot be ignored because the passed distance and the load carried are significantly different between vehicles. In order to yield better results, it should be optimized through MOPSO algorithm.

Table 1. Input values

\begin{tabular}{|c|c|c|c|}
\hline Demand & & $y$ & $\mathrm{x}$ \\
\hline & Depot & 25 & 55 \\
\hline 442 & Customer 1 & 62 & 14 \\
\hline 611 & Customer 2 & 47 & 15 \\
\hline 168 & Customer 3 & 35 & 26 \\
\hline 148 & Customer 4 & 83 & 84 \\
\hline 578 & Customer 5 & 59 & 25 \\
\hline 802 & Customer 6 & 55 & 82 \\
\hline 941 & Customer 7 & 82 & 24 \\
\hline 217 & Customer 8 & 28 & 93 \\
\hline 612 & Customer 9 & 76 & 35 \\
\hline 522 & Customer 10 & 76 & 19 \\
\hline \multicolumn{2}{|c|}{ Capacity } & \multicolumn{2}{|c|}{ Vehicle } \\
\hline \multicolumn{2}{|c|}{2022} & \multicolumn{2}{|c|}{ Vehicle 1} \\
\hline \multicolumn{2}{|c|}{2186} & \multicolumn{2}{|c|}{ Vehicle 2} \\
\hline \multicolumn{2}{|c|}{2098} & \multicolumn{2}{|c|}{ Vehicle 3} \\
\hline
\end{tabular}

\section{Optimized results}

In this case, three repetitions were also considered for simulation and the results were compared.

In the first state, which is completely feasible, the movement route of each vehicle is close to the other ones and the capacity is almost equal. Additionally, it can be seen that after 47 repetitions the output became stable and the best results were obtained. In the second state, it can also be seen that the results are feasible and after 69 repetitions, the output became stable and the best results were obtained. In the last state, the results are feasible; after 21 repetitions, the output became stable and the best results were obtained.

\section{CONCLUSION}

Finding the routes of vehicles is a logistic issue, which has recently been investigated in many studies, and typically, the purpose of a vehicle routing problem is to find a set of routes for several vehicles from one depot to a number of customers and returning to the depot without violation of capacity limit of each vehicle with minimum cost. The combination of customers is not limited to the choice of transportation routes; VRP is considered as a combinative optimization problem, in which the number of possible responses to the problem is increased exponentially along with the number of customers. Today, with the development of industries and emergence of competitive markets, managers of industrial and service centers are seeking to reduce the operational costs and thus reduce the end cost of products and services. Reducing the transportation costs can be very important in saving costs. One of the issues in the field of optimizing transportation costs is the vehicle routing. Time-dependent vehicle routing is one type of dynamic vehicle routing. This issue, in its basic form, is a problem of routing vehicles that are distributed in a fleet of vehicles from one or more central depots in order to serve a set of customers and, upon completion of operations, return to the relevant depot. The main goal of this is to minimize total travel time and its resulting costs. Under these conditions, the travel time is divided between customers as well as between customers and depot, and consequently, the travel costs are dependent on the time period during which the travel is done. Increasing the efficiency and performance of transportation 

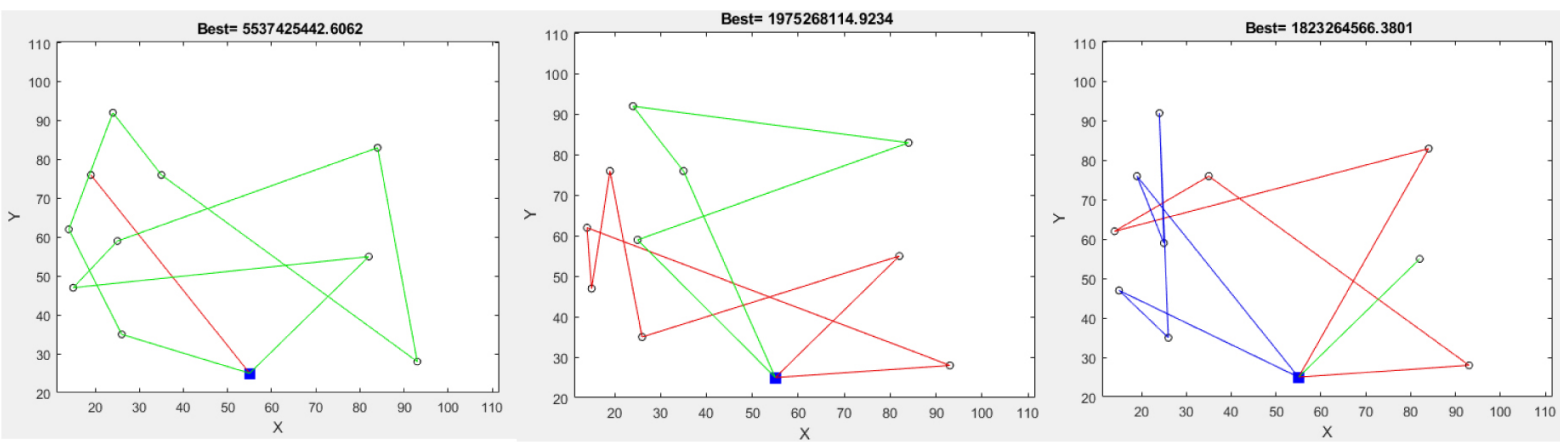

Figure 2. Routing before optimization, A) first repetition, B) second repetition, C) third repetition

Table 2. Results of consecutive repetitions before optimization

\begin{tabular}{|c|c|c|c|c|c|c|c|c|c|c|c|c|}
\hline \multicolumn{2}{|c|}{ Vehicle } & \multirow{2}{*}{$\frac{\text { Distance }}{124.851912}$} & \multirow{2}{*}{$\begin{array}{c}\text { Used capacity } \\
522\end{array}$} & \multicolumn{9}{|c|}{ Customer } \\
\hline First & Vehicle 1 & & & 10 & & & & & & & & \\
\hline repetition & Vehicle 2 & 429.431058 & 4519 & 3 & 1 & 7 & 9 & 8 & 4 & 5 & 2 & 6 \\
\hline \multirow{2}{*}{$\begin{array}{l}\text { Second } \\
\text { repetition }\end{array}$} & Vehicle 1 & 309.850325 & 2762 & 8 & 1 & 2 & 10 & 3 & 6 & & & \\
\hline & Vehicle 2 & 243.906837 & 2279 & 5 & 4 & 7 & 9 & & & & & \\
\hline \multirow{3}{*}{$\begin{array}{l}\text { Third } \\
\text { repetition }\end{array}$} & Vehicle 1 & 276.571334 & 1419 & 8 & 9 & 1 & 4 & & & & & \\
\hline & Vehicle 2 & 80.7217443 & 802 & 6 & & & & & & & & \\
\hline & Vehicle 3 & 232.433607 & 2820 & 2 & 3 & 7 & 5 & 10 & & & & \\
\hline
\end{tabular}

systems is one of the most important issues that need to be addressed, so that we can face less traffic and prevent cost increase; thus, vehicles routing problem (VRP) has emerged. In fact, the vehicles routing problem refers to a set of problems in which a number of vehicles concentrated in one or more locations should go to a set of customers and provide a service that each has a specific demand. Vehicle Routing Problem Time Window (VRPTW) is a generalized issue of Capacity Vehicle Routing Problem (CVRP), in which the service provided to each customer should be made within a given time period, known here as the time window. Many companies are faced with this problem every day.

Routing refers to finding an ideal route that meets the model objectives and complies with the customer, while through planning the vehicles, the time that each customer must be served is determined. Accordingly, the issue of total time and cost of the route not only includes the total cost of the distance and service time and other related costs, but the total cost of stops and waiting is taken into account, too. Urban environments have a great structural complexity. This has enabled access to different points through more than one route. Under such conditions, traffic regulations for routes, such as determining maximum permitted speed and traffic constraints on transportations vehicles affect the choice of the route to continue the travel through vehicles. Choosing the appropriate route can be very important for distribution companies and rental transportation companies, especially when receiving orders at a specific time is important for customers, and they can be optimized and selected through different algorithms. Therefore, in the present study we provided a model for routing motion of vehicles with a specific origin using a meta-heuristic algorithm, and we optimized the problem of providing a routing model. The transportation issues today are of interest to most researchers and relevant authorities, because solving this problem is of great help in many areas. The transportation cost is an effective component in the end price of goods as well as customer's satisfaction level. VRP has been dealt with since the 1960s, and various methods have been presented to solve it. Developments, such as open vehicle routing problem, vehicle routing problem along with simultaneous receiving and delivery of goods, vehicle routing problem along with time windows etc. were presented. This topic is not restricted to a few university disciplines that utilize it only for traffic management, but it also includes all domains. Therefore, addressing this issue is very important for correctly solving the problem of vehicles transportation with time window. What is recommended in this research as suggestions 

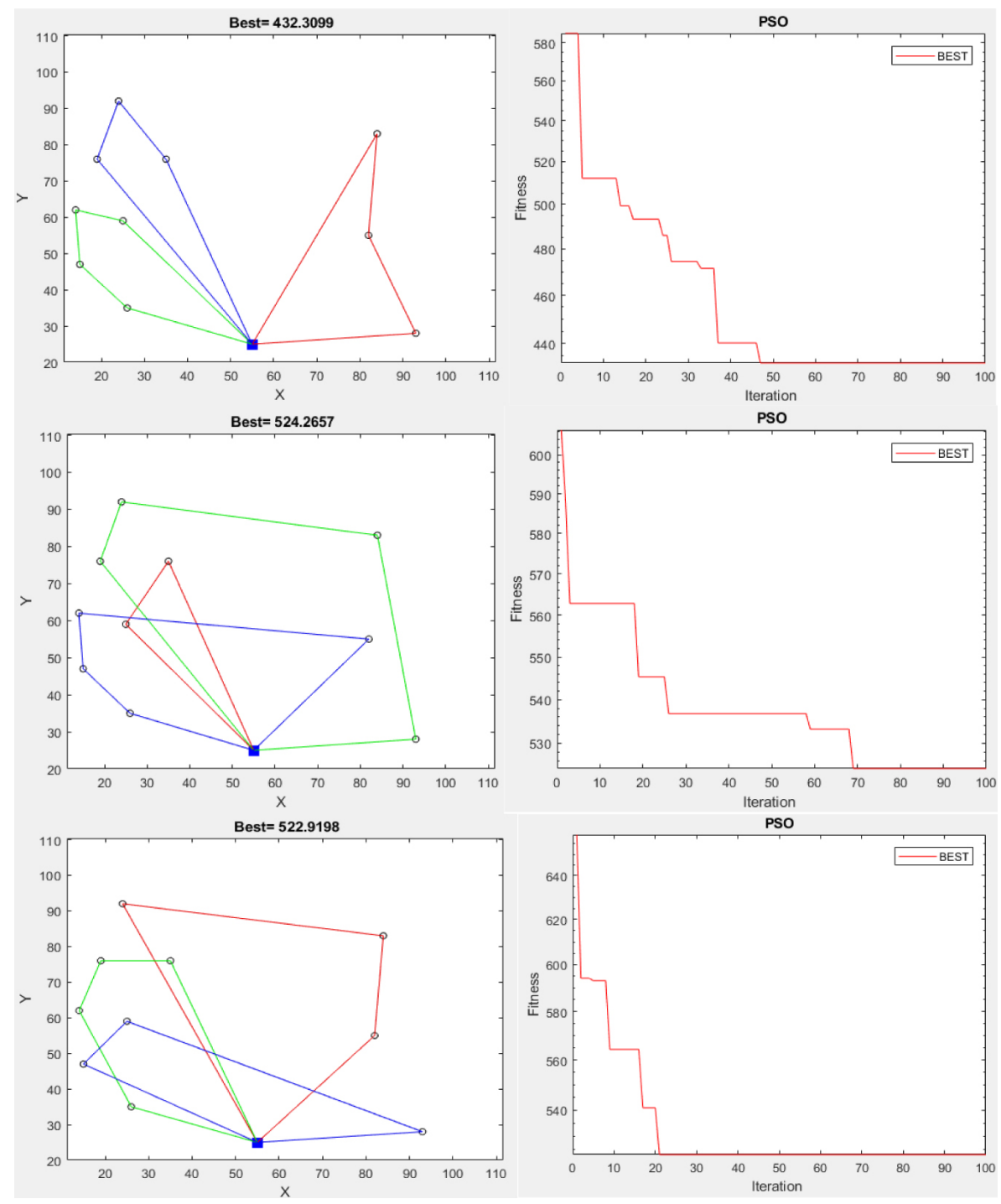

Figure 3. Routing after optimization and convergence of algorithm, A) first repetition, B) second repetition, C) third repetition

Table 3. Results of consecutive repetitions after optimization

\begin{tabular}{|c|c|c|c|c|c|c|c|c|c|c|c|c|}
\hline \multicolumn{2}{|c}{ Vehicle } & Used capacity & Distance & \multicolumn{7}{c|}{ Customer } \\
\hline \multirow{4}{*}{$\begin{array}{c}\text { First } \\
\text { repetition }\end{array}$} & Vehicle 1 & 160.190306 & 1167 & 8 & 6 & 4 & & & & & & \\
\hline & Vehicle 2 & 118.732731 & 1799 & 3 & 2 & 1 & 5 & & & & & \\
\hline & Vehicle 3 & 153.386882 & 2075 & 10 & 7 & 9 & & & & & & \\
\hline \multirow{3}{*}{$\begin{array}{c}\text { Second } \\
\text { repetition }\end{array}$} & Vehicle 1 & 119.847603 & 1190 & 5 & 9 & & & & & & & \\
\hline & Vehicle 2 & 233.709992 & 1828 & 8 & 4 & 7 & 10 & & & & & \\
\hline & Vehicle 3 & 170.708057 & 2023 & 3 & 2 & 1 & 6 & & & & & \\
\hline \multirow{3}{*}{$\begin{array}{c}\text { Third } \\
\text { repetition }\end{array}$} & Vehicle 1 & 202.92757 & 1891 & 7 & 4 & 6 & & & & & & \\
\hline & Vehicle 2 & 145.869749 & 1744 & 9 & 10 & 1 & 3 & & & & & \\
\hline
\end{tabular}

for future research is to study the routing problem by integrating various meta-heuristic algorithms and comparing their results. The research can also be developed and expanded by applying conditions such as simultaneous delivery and loading of goods, presentation of a model for corruptible goods, application of scarcity and discount states, repetition of the model for heterogeneous vehicles and use of lateral transfer approach between demand centers. 


\section{REFERENCES}

1. Rahmani H.A., Asghar A. and Kiani K., (2016), Using improved gravity algorithm for solving open vehicle routing problem. Second International Conference on New Research Findings in Electrical Engineering and Computer Sciences, Kasra NonProfit Institution of Higher Education, Ramsar.

2. Sattak M., Habibi M., Karimi H. and Abedzadeh M., (2013), Modeling and solving time-dependent vehicle routing problem with semi-soft Time Windows in Multiple Graphs; Transportation Research Journal. 10(3).

3. Sargardan F., Kia V.R. and Ghaffari M., (2015), Modeling vehicle routing problem considering multiple depots, simultaneous delivery and loading, hard and soft time window, cost and depreciation depending on the amount of load existing on the vehicle and the route type. International Conference on New Researches in Management and Industrial Engineering, Tehran, Modiran Ide Parazan Paytakht Ilia Co.

4. Salamatbakhsh, A., Moghaddam R.T. and Nowroozi N., (2016), Open vehicle routing problem considering drivers' satisfaction: multi-objective evolutionary algorithm based on analysis. Quarterly of Transport Engineering, 7(3).

5. Yousefi Khoshbakht M. and Rahmati F. (2011), An improved algorithm for ants population for solving vehicle routing problem, along with simultaneous receiving and delivering of goods. Quarterly of Transportation, 8th Year, 2, 183-198.

6. Chen, H.K., Hsueh, C.F. and Chang, M.S. (2006) The real-time time-dependent vehicle routing problem. Transportation Research Part E, 42, 383-408.
7. Hashimoto, H., Yagiura, M., and Ibaraki, T. (2008), An iterated local search algorithm for the timedependent vehicle routing problem with time windows. Discrete Optimization, 5, 434-456

8. Kok, A.L., Hans, E.W., and Schutten, J.M.J. (2012), Vehicle routing under time-dependent travel times: the impact of congestion avoidance. Computers \& Operations Research, 39(5), 910-918.

9. Kok, A.L., Hans, E.W., Schutta, J.M.J. and Zijm, W.H.M. (2010), A dynamic programming heuristic for vehicle routing with time-dependent travel times and required breaks. Flexible Services and Manufacturing Journal, 22, 83-108.

10. Kumar, M, Narasimha, K.V., Kivelevitch, E., Sharma, B. (2013), An ant colony optimization technique for solving min-max multi-depot vehicle routing problem. Swarm and Evolutionary Computation, 13, 63-73.

11. Kuo, Y. (2010), Using simulated annealing to minimize fuel consumption for the timedependent vehicle routing problem. Computers \& Industrial Engineering, 59, 157-165.

12. Kuo, Y., Wang, C.C. and Chuang, P.Y. (2009), Optimizing goods assignment and the vehicle routing problem with time-dependent travel speeds.Computers \& Industrial Engineering, 57, 1385-1392

13. Schneider, M., Stenger, A. and Goeke, D. (2014), The electric vehicle-routing problem with time windows and recharging stations. Transportation Science, 48(4), 500-520.

14. Wang, Z. and Wang, Z. (2009), A novel two-phase heuristic method for vehicle routing problem with backhauls. Computers and Mathematics with Applications, 57, 1923-1928. 